\title{
Capabilities and limitations of 3D printed microserpentines and integrated 3D electrodes for stretchable and conformable biosensor applications
}

\author{
Charles Didier $\mathbb{D}^{1,2}$, Avra Kundu ${ }^{1}$ and Swaminathan Rajaraman 1,2,3,4
}

\begin{abstract}
We explore the capabilities and limitations of 3D printed microserpentines ( $\mu$ serpentines) and utilize these structures to develop dynamic 3D microelectrodes for potential applications in in vitro, wearable, and implantable microelectrode arrays (MEAs). The device incorporates optimized 3D printed $\mu$ serpentine designs with out-of-plane microelectrode structures, integrated on to a flexible Kapton ${ }^{\circledR}$ package with micromolded PDMS insulation. The flexibility of the optimized, printed $\mu$ serpentine design was calculated through effective stiffness and effective strain equations, so as to allow for analysis of various designs for enhanced flexibility. The optimized, down selected userpentine design was further sputter coated with 7-70 nm-thick gold and the performance of these coatings was studied for maintenance of conductivity during uniaxial strain application. Bending/conforming analysis of the final devices (3D MEAs with a Kapton ${ }^{\circledR}$ package and PDMS insulation) were performed to qualitatively assess the robustness of the finished device toward dynamic MEA applications. 3D microelectrode impedance measurements varied from 4.2 to $5.2 \mathrm{k} \Omega$ during the bending process demonstrating a small change and an example application with artificial agarose skin composite model to assess feasibility for basic transdermal electrical recording was further demonstrated.
\end{abstract}

\section{Introduction}

Stretchable electronics and microsensors have begun to be applied to several consumer and biomedical areas, including wearables for personal health monitoring ${ }^{1,2}$, surgical robotics ${ }^{3}$, implantable devices ${ }^{4}$, tactile sensors ${ }^{5}$, and devices for power harvesting and storage ${ }^{6}$. A basic requirement in the micro-structuring of such devices is the design and development of the components of the system that are able to mechanically deform without losing their ability to electrically function successfully. Inorganic materials used in the microfabrication of

Correspondence: Swaminathan Rajaraman (Swaminathan.Rajaraman@ucf.edu) ${ }^{1}$ Nanoscience Technology Center (NSTC), University of Central Florida, Orlando, FL 32826, USA

${ }^{2}$ Burnett School of Biomedical Sciences, University of Central Florida, Orlando, FL 32827, USA

Full list of author information is available at the end of the article. stretchable microsensors such as silicon ${ }^{7}$ and aluminum ${ }^{8}$ are very stiff and deform to an extent where electrical failure occurs at small amounts of tensile strain ${ }^{9}$. In order to alleviate this problem, a common strategy for a device design with such materials, is to replace "straight wire" features ${ }^{10}$ fabricated out of these materials with shapes engineered to be stretchable and flexible including "Archimedean spiral" ${ }^{\text {"11 }}$ " $\mu$ serpentines," and other geometries $^{6,12}$. Specifically in flexible electronics devices, "serpentine" designs have resulted in enhanced strain performance ${ }^{13}$. In addition to the aforementioned standard materials, there are numerous material sets and combinations currently in use for the fabrication of stretchable electronics, with polydimethylsiloxane (PDMS) being a widely used substrate and packaging material $^{14,15}$.

\section{(c) The Author(s) 2020}

(c) (i) Open Access This article is licensed under a Creative Commons Attribution 4.0 International License, which permits use, sharing, adaptation, distribution and reproduction c. in any medium or format, as long as you give appropriate credit to the original author(s) and the source, provide a link to the Creative Commons license, and indicate if changes were made. The images or other third party material in this article are included in the article's Creative Commons license, unless indicated otherwise in a credit line to the material. If material is not included in the article's Creative Commons license and your intended use is not permitted by statutory regulation or exceeds the permitted use, you will need to obtain permission directly from the copyright holder. To view a copy of this license, visit http://creativecommons.org/licenses/by/4.0/. 
A common structure in a stretchable electronics system is microelectrode, which consists of a substrate (with an additional package or the package defined on the substrate) atop which a grid or line of metal traces and an insulation layer are defined ${ }^{16}$. Such two-dimensional (2D) and three-dimensional (3D) microelectrode arrays (MEAs) have become ubiquitous in in vitro, cell-based biosensing $^{17}$, wearable ${ }^{18}$, implantable ${ }^{19}$, and environmental sensing applications ${ }^{20}$.

Recently, the ease of microfabrication of complex shapes such as $\mu$ serpentines and base structures for $2 \mathrm{D}$ and 3D MEAs has been achieved through rapid and costeffective additive manufacturing methods like $3 \mathrm{D}$ printing $^{1}$. Owing to the commercial availability of various 3D printing systems and the innovations of makerspace environments, the development of 3D printed devices has increasingly expanded and continues to show promise in innovation $^{21}$. While prior work demonstrating the development of 2D and 3D MEAs in static cell culture settings has been reported ${ }^{22}$ (including from our group), to date understanding the capabilities and limitations of 3D printed geometries and their application to stretchable and dynamic 3D microelectrodes is missing.

In this work, considerations and limitations for using standard and commercially available clear $\operatorname{resin}^{23}$ to produce a stretchable and flexible engineered design that can incorporate robust $3 \mathrm{D}$ structures through additive micro-stereolithographic ( $\mu \mathrm{SLA}) 3 \mathrm{D}$ printing is explored. This work has adapted and expanded on the mathematical background on printed $\mu$ serpentine structures that was recently developed ${ }^{24}$. Similarly, the metallization of such 3D printed structures has not been fully characterized or understood. To this end, metallized 3D printed $\mu$ serpentines were analyzed for performance, reliability, and bending/conformance.

Beyond the optimization of metallized $\mu$ serpentines, 3D MEA devices still require a package and an insulation. Materials such as polyimide (PI) and PDMS provide choices in polymeric backbone layers with improved mechanical match for dynamic biological tissue experiments (Young's modulus of PI: $2.5 \mathrm{GPa}^{25}$ and PDMS: $360 \mathrm{kPa}-2.97 \mathrm{MPa}^{26,27}$ ). Further, PDMS is commonly used in $2-2.5 \mathrm{D}$ flexible devices as both the substrate and the insulation material, because it also provides tunable mechanical and dielectric properties ${ }^{28}$. In this work, PDMS has been used as an elastomeric insulation and thin Kapton ${ }^{\circledR}$ PI has been used as a packaging substrate.

A schematic for the microfabrication and packaging of the 3D MEAs is depicted in Fig. 1a. The stretchable, conformable 3D MEA was constructed with a singular $\mu$ serpentine design and was subsequently electrically and mechanically characterized. An analytical model was developed for the design of the printed $\mu$ serpentine structures. Further, to demonstrate the potential applicability of this device in biopotential measurements, puncture and conductivity characteristics on an artificial skin agarose model were explored. Such a technology platform introduces a novel, flexible, rapidly fabricated,

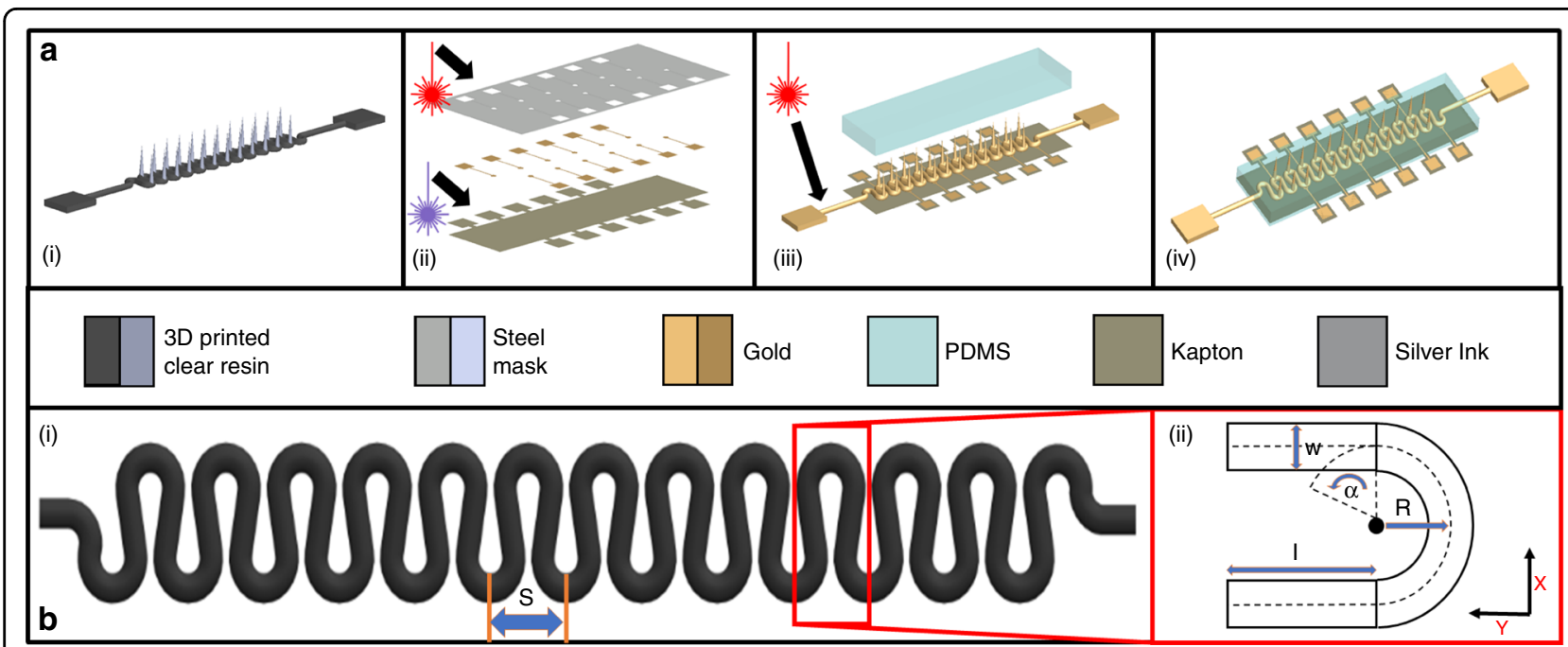

Fig. 1 Schematic representation of the process flow for the dynamic 3D MEA fabrication with $\mu$ serpentines and details of the userpentines. a Schematic of the process flow. (i) 3D printed $\mu$ serpentine with out-of-plane electrode structures. (ii) Initial fabrication steps, including the UV laser micromachining of the Kapton ${ }^{\circledR}$ substrate and the IR laser micromachining of the steel deposition mask with associated sputter metallization of the Au traces. (iii) Assembly of the full device, where a metallized $\mu$ serpentine is IR laser micromachined selectively to isolate the electrodes and then is placed on the Kapton package and insulated with PDMS. (iv) Schematic of the fully assembled device. $\mathbf{b}$ Schematic of a userpentine for illustration of the geometric parameters. (i) $\mu$ serpentine denoting a singular "S" subunit and highlighting (ii) illustrates the various geometric parameters and reference orientation. 
and cost-effective packaging substrate that can be applied to a variety of flexible biosensor, wearable, implantable, and cell/tissue culturing applications ${ }^{2,29}$.

\section{Results and discussion}

Widlund et al. compiled the two key equations, which laid the foundation for analytically modeling $\mu$ serpentine geometries and down selecting 3D printed test structures $^{24}$. Their work combined and condensed plane-strain elastic theory ${ }^{30}$, as well as Winkler curved beam theory ${ }^{31}$ to produce Eqs. 1 and 2 as outlined below.

Equation 1 is the compound equation for the effective stiffness of a given $\mu$ serpentine design:

$$
\begin{aligned}
& \frac{\mathrm{PS}}{2 \overline{\mathrm{E}} w u_{0}}= \frac{\left(\frac{w}{R}\right) *\left((\operatorname{Cos}[\alpha])-\left(\left(\frac{l}{(2 R)}\right) \operatorname{Sin}[\alpha]\right)\right)}{2\left[\left(\left(\operatorname{Cos}[\alpha]^{2}\right)\left(\left(\frac{l^{3}}{\left(2 R^{3}\right)}\right)+\left(\left(3\left(\frac{\pi}{2}+\alpha\right)\right)\left(\frac{l^{2}}{R^{2}}\right)\right)+\left(12\left(\frac{l}{R}\right)\right)-12\left(\frac{\pi}{2}+\alpha\right)\right)\right)\right.} \\
&+\left(\operatorname{Sin}[2 \alpha]\left(\left(6\left(\frac{\pi}{2}+\alpha\right)\left(\frac{l}{R}\right)+9\right)\right)\right)
\end{aligned}
$$

In this equation, " $P$ " denotes the reaction force, " $S$ " is the length of a given serpentine, " $w$ " is the width of that serpentine, and " $2 u_{0}$ " is the effective displacement of the serpentine, giving rise to $\mathrm{PS} /\left(2 \overline{\mathrm{E}} w u_{0}\right)$ on the left side of the equation, which is expanded on the right side as determined in the work by Widlund et al. ${ }^{24}$.

Equation 2 is similarly the compound equation for the maximum effective strain on the inner U-bend curvature (Fig. $2 \mathrm{~b}$ ) of a given $\mu$ serpentine design and is expanded from $\varepsilon_{\max }$ (the maximum tensile strain on the serpentine) and $\varepsilon_{\text {applied }}$ (the effective applied tensile strain): denoted as "Ē" and the shear modulus of the resin material is denoted as "G." These values were calculated using the Young's modulus of the Formlabs Form 2 Clear resin at $2.8 \mathrm{GPa}^{23}$ and Poisson's ratio of 0.4 (poly(methyl methacrylate) $)^{32}$, which is the closest approximation available for the resin, since it is largely proprietary but is known to be methacrylate based ${ }^{23}$.

The $\alpha$ values denote the degree of completeness of the central arc of the semi-circle of the $\mu$ serpentine U-bend, with respect to a standard semi-circle (has an $\alpha$ value of $0^{\circ}$ ). All values of $\alpha$ are denoted in one quadrant of the central circular arc length and are reflected bilaterally

$$
\begin{gathered}
\frac{\varepsilon[\max ]}{\varepsilon[\operatorname{app}]}=\frac{\left(\left(\frac{w}{R}\right)\left(\left(\frac{12}{\left(2-\left(\frac{w}{R}\right)\right)}\right)+\left(\left(\left(\frac{12}{\left(2-\left(\frac{w}{R}\right)\right)}\right)-\left(\frac{w}{R}\right)\right)\left(\operatorname{Sin}[\alpha]+\left(\left(\frac{l}{2 R}\right) \operatorname{Cos}[\alpha]\right)\right)\right)\right) *\left(\left(\operatorname{Cos}[\alpha]-\left(\left(\frac{l}{2 R}\right) \operatorname{Sin}[\alpha]\right)\right)\right)\right)}{\left(\left(\operatorname{Cos}[\alpha]^{2}\right)\left(\left(\frac{l^{3}}{\left(2 R^{3}\right)}\right)+\left(\left(3\left(\frac{\pi}{2}+\alpha\right)\right)\left(\frac{l^{2}}{R^{2}}\right)\right)+\left(12\left(\frac{l}{R}\right)\right)-12\left(\frac{\pi}{2}+\alpha\right)\right)\right)} \\
+\left(\operatorname{Sin}[2 \alpha]\left(\left(6\left(\frac{\pi}{2}+\alpha\right)\left(\frac{l}{R}\right)+9\right)\right)\right)+\left(\left(\left(\left(\frac{w^{2}}{R}\right)\left(\frac{\pi}{2}+\alpha\right)\left(\frac{l}{(2 R)} \operatorname{Cos}[\alpha]+\operatorname{Sin}[\alpha]\right)^{2}\right.\right.\right. \\
\left.\left.\left.+\left(\left(\frac{l}{2 R}\right)\left(\operatorname{Sin}[\alpha]+\left(\left(\frac{3 \overline{\mathrm{E}}}{2 G}\right) \operatorname{Cos}[\alpha]\right)\right)\right)\right)+\left(18\left(\frac{\pi}{2}\right)+\alpha\right)\right)\right)
\end{gathered}
$$

Both equations were tabulated with respect to the specific aspects of a $\mu$ serpentine interconnect geometry, as shown in Fig. $1 \mathrm{~b}$ (ii). In these equations, close attention needs to be devoted to the $\alpha$ value and the ratio of $l / R$.

The resin used for the device in this work was the Clear (FLGPCL04) resin from Formlabs ${ }^{23}$. This material is inherently inflexible but could resolve the necessary structures to create 3D electrodes to be used in the final device and as a result was chosen as the material for 3D printing. The plane strain modulus of the resin material is across the semi-circle. The positive values of $\alpha$ denote a circle closer to completion. An $\alpha$ value of $0^{\circ}$ being a standard semi-circle and an $\alpha$ of $90^{\circ}$ being a completed circle. Values of $\alpha<0^{\circ}$ denote a less than complete circle, and thus the structure would reach a flat and straight ribbon at an $\alpha$ of $-90^{\circ}$. The length " $l$ " is the distance between the U-bends of the $\mu$ serpentine interconnects, and the ratio of this length to the radius " $R$ " of the Ubend's semi-circle, is an important distinction for the distribution of strains as the $\mu$ serpentine is stretched. The width " w" was fixed for this analytical calculation at an experimentally defined value of $400 \mu \mathrm{m}$. This was the smallest dimension that the Form $2 \mu$ SLA 3D printer could resolve in this configuration because of the need for printing support structures. Similarly, the value of " $R$ " was fixed at $400 \mu \mathrm{m}$ to ensure maximum printability given the previous width constraint. It should be noted, that because of the $\mu$ serpentine's circular profile in this experimental set-up, the thickness and the width were set as equivalent, which differs from the original mathematical set-up of Widlund et al. ${ }^{24}$, in which the $\mu$ serpentines 
were printed to have a rectangular cross-sectional profile, leading to differing width and thickness. The singular subunit for a given $\mu$ serpentine design is also illustrated in Fig. $1 b(i)$, as denoted by "S."

Figure 2a, b, shows a contour plot of Eqs. 1 and 2. These contour plots represent the variation of normalized stiffness and $\varepsilon_{\max } / \varepsilon_{\text {applied, }}$, where the angle $\alpha$ was varied along the $x$ axis, and the ratio of $l / R$ varied on the $y$-axis. In both the graphs, the quantity $w / R$ was fixed for calculations because the width of the $\mu$ serpentine never changed owing to the experimental constraint previously mentioned. Figure 2a was normalized with respect to the calculated values of the expected stiffness (Eq. 1) for an $\alpha$ of $-90^{\circ}$, where the $\mu$ serpentine would devolve into a flat ribbon and thus would be the stiffest conformation. Figure $2 \mathrm{~b}$ is a similarly derived contour plot representing analytical calculations from Eq. 2, which denotes the maximum effective strain that would be applied to the inner curvature of the U-bends. The values denoted in white in this figure violate the "non-overlapping constraint" as outlined in Widlund et $\mathrm{al}^{24}$, which represent designs that are mathematically and geometrically impossible. Printing at the smallest resolvable design conformation on a $\mu$ SLA 3D printer has associated design challenges, which limit combinations of $l / R$ and $\alpha$ that were chosen in this work. The region in Fig. 2a corresponding to a normalized stiffness between 0 and 0.25 represents the design conformations of a possible $\mu$ serpentine, which would be the least stiff. While this might appear advantageous, theoretical and $\mu$ SLA print constraints (including more scaffolding supports for longer " $l$ " values) necessitate design choices that balance stiffness and effective strain.

Three designs were chosen and are denoted on the graph with black circles in Fig. 2a, b. All three of the variants had $l /$ $R$ ratios of 2 (with $l=800 \mu \mathrm{m}$ ) in order to ensure nonoverlapping print configurations, as higher values of " $l$ " would have resulted in fused $\mu$ serpentines at $\alpha=10^{\circ}$. As previously mentioned, there is a mutually exclusive relationship between the supporting scaffolds necessary to resolve $\mu$ serpentines and $\mu$ serpentines with a lower stiffness value. A minimum of one linear support per " $S$ " unit along the $x$-axis (denoted in Fig. $1 \mathrm{~b}(\mathrm{ii}))$ is necessary to resolve the $l / R=2$ designs. Increasing the " $l$ " value would increase

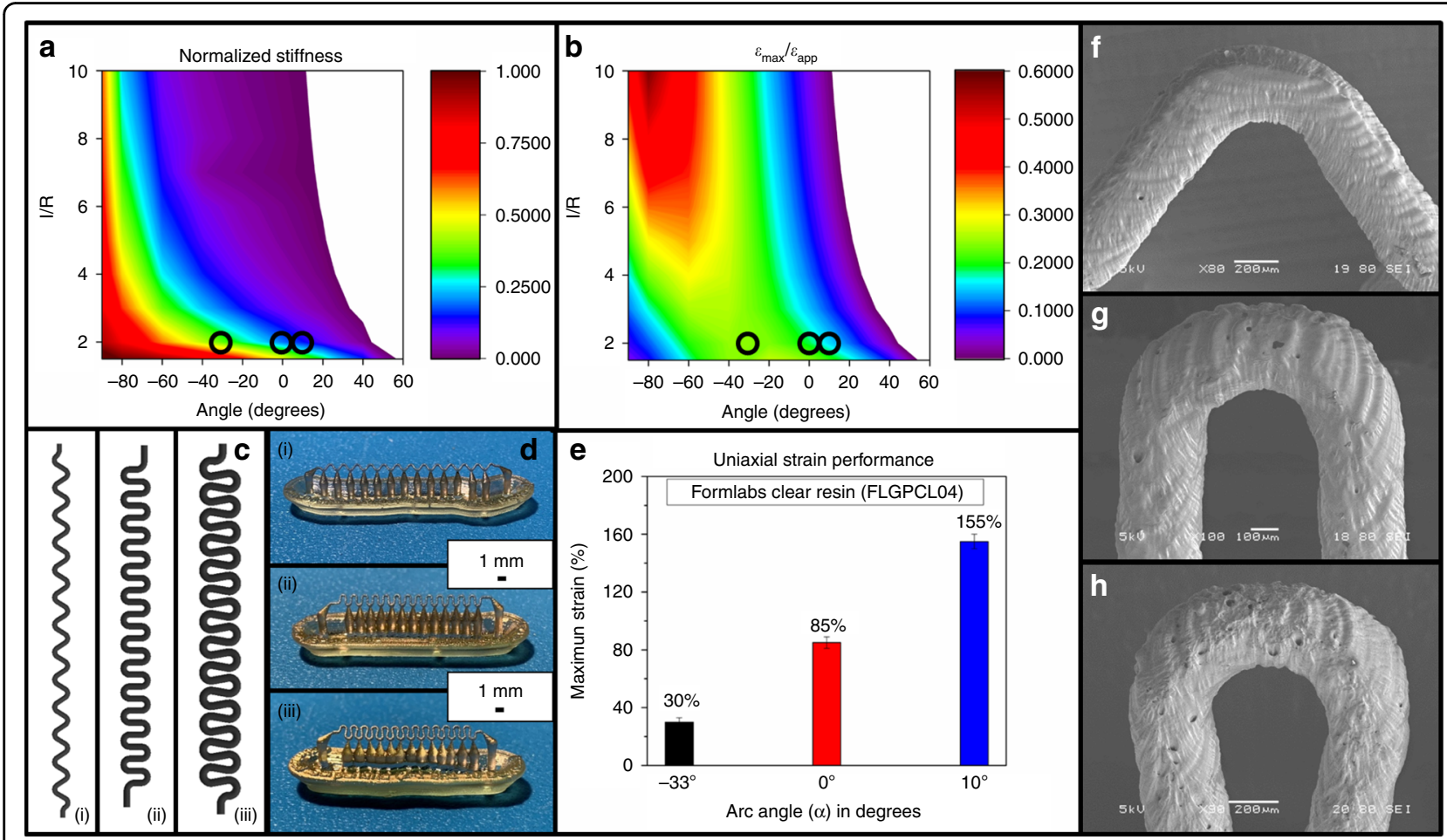

Fig. 2 Graphical representations of the $\mu$ serpentine modeling equations and simple strain data, with images of the printed $\mu$ serpentines. a Contour plot of the normalized stiffness for a $\mu$ serpentine (with Formlabs Clear resin) calculated from the analytical model developed using Eq. 1. The hotter colors denote conformation closer to the stiffness of a flat ribbon (where $a=-90^{\circ}$ ). Circles indicate the design choices for this work. $\mathbf{b}$ Contour plot of the maximum effective strain on the inner U-bend of a $\mu$ serpentine, calculated from the analytical model developed using Eq. 2. The lower values in this contour plot indicate a higher maximum effective strain that can be applied before failure. Circles indicate design choices for this work. $\mathbf{c}$ (i-iii) Schematic representations of the three chosen $\mu$ serpentine designs, from $a=-33^{\circ}$ (i), to $a=0^{\circ}$ (ii), and $a=10^{\circ}$ (iii). $\mathbf{d}$ Optical micrographs of the $\mu S L A 3 D$ printed $\mu$ serpentines after metallization corresponding to their schematics in $\mathbf{c}$. e Experimentally measured maximum uniaxial strain of the three $\mu$ serpentine designs, leading to the down selection of the $a=10^{\circ}$ design. $\mathbf{f}-\mathbf{h}$ SEM images of the three $\mu$ serpentine designs corresponding to (i-iii) in c. The minor print defects seen from the $\mu S L A$ printing process do not impact the design's performance and are consistent across all prints. 
scaffolding necessary for a " $S$ " unit along the $y$-axis, to values $>1$. This would result in either fused prints or unstable printed structures, negating benefits that are theoretically possible. This limitation narrowed down the real variation in design stretchability and flexibility to the value of $\alpha$, which in fact does contribute greatly to the overall performance of a particular design. Values for $\alpha$ at $0^{\circ}$ and $10^{\circ}$ were chosen to illustrate (according to the calculated theoretical data) that the small increase of $10^{\circ}$ would significantly have a positive impact on the design (supported in Fig. 2b). The $\alpha=-33^{\circ}$ was arbitrarily chosen in the negative region to be closer to the mid-range of stiffness values and to study negative design values of $\alpha$. These decisions are supported by the analytical model for maximum strain which shows that these values for the inner U-bends are inversely related to the overall maximum stretchability of the $\mu$ serpentine design $^{24}$. Figure $2 c(i-i i i)$ demonstrate the 3D CAD renderings of the three chosen $\mu$ serpentine designs, and Fig. $2 \mathrm{~d}$ (i-iii) illustrate the optical micrographs of the 3D printed and metallized designs before release from their printing support structures. Figure 2e plots the effective maximum strain attained by the three designs experimentally (average $N=6$ ). The $\alpha=-33^{\circ}$ design had the poorest performance with failure at $30 \%$ increase in length during uniaxial strain testing. The $\alpha=0^{\circ}$ design performed largely better as expected, with the ability to extend up to a maximum of $85 \%$ additional strain from rest prior to failure.

The optimized design $\left(\alpha=10^{\circ}\right)$ as suggested by the analytical model and expected to outperform the other two designs was able to resist failure until a uniaxial strain of $155 \%$ was applied to the structure. This structure was chosen for further metallization optimization and device fabrication. Figure 2f, g shows scanning electron microscopic (SEM) images of the three designs, after printing. The striations and small defects in the resin surface are standard features of $\mu S L A$ printing, by virtue of the laser-spot definition of individual printed layers, and did not have any significant impact on the performance of the $\mu$ serpentine structures.

To accomplish conductive $\mu$ serpentines toward the goal of 3D stretchable microelectrodes, a conformal metal deposition technique was needed that could coat the striated surface of the $\mu$ serpentine and maintain its integrity under strain. Sputter metal coating provides an ideal, easily accessible method to accomplish conformal metal coatings with precisely defined thicknesses ${ }^{33}$. Five different coating thicknesses $(7,14,20,33$, and $70 \mathrm{~nm})$ were assessed to obtain an optimal coating of sputtered gold, with resistance performance under strain as the measurand. Figure $3 a-e$ shows the single cycle hysteresis for each of the five coating thicknesses, where the maximum strain value for the optimized $\alpha=10^{\circ}$ design never exceeded $100 \%$ uniaxial strain (well below the maximum value of $155 \%$, Fig. 2e). The change in resistance to resistance at rest $(\Delta R / R)$ was calculated to see the reliability of each of the coatings during a single stretch cycle (Fig. 3a-e). Figure $3 \mathrm{f}$ shows a plot of the $\Delta R / R$ values from the previous graphs (Fig. $3 \mathrm{a}-\mathrm{e}$ ), and highlights that asymptotic region encompassing the 20,33 , and $70 \mathrm{~nm}$ coatings. This low variance in the resistance values indicates that this asymptotic range is the most suitable for consistent conduction values while the device is under strain. This is evident when examining the requirements for viable $\mu$ serpentine strain sensors, which require a much higher $\Delta R / R^{34}$. The inset to Fig. 3f corroborates this finding, as the integrated $\Omega(\Delta l) / l$ under the hysteresis curve are lowest for these three coatings as well: $7 \mathrm{~nm}$ corresponding to an integrated area of $56,070 \Omega(\Delta l) / l$, $14 \mathrm{~nm}$ with an area of $13,659.84 \Omega(\Delta l) / l, 20 \mathrm{~nm}$ with an area of $9,565 \Omega(\Delta l) / l, 33 \mathrm{~nm}$ with an area of $832 \Omega(\Delta l) / \mathrm{l}$, and $70 \mathrm{~nm}$ with an area of $1,004.5 \Omega(\Delta l) / l$. This indicates a much tighter strain to relaxation behavior for the thicker coatings, which would improve device stability and reliability over time. The $33 \mathrm{~nm}$ has the lowest area under such a hysteresis curve and was chosen as the most reliable value for device construction.

To analyze fracture compositional changes in the coatings after the application of strain, energy dispersive $\mathrm{X}$-ray spectroscopy (EDS) was performed on the coatings with the lowest area under such a hysteresis curve. Figure 4 depicts SEM images of the $20 \mathrm{~nm}$ (Fig. 4a), $33 \mathrm{~nm}$ (Fig. 4b), and $70 \mathrm{~nm}$ (Fig. 4c) U-bends and the associated fractures in the gold film from applied strain, after being subjected to one-cycle strain and relaxation. Each SEM has two highlighted sections for compositional comparison using EDS: the red circle denotes regions inside the fracture, and blue circle denotes regions in the unaffected gold film outside the fracture. The focus of the fracture study was placed on the inner curvature of the U-bend as informed by the analytical model since these areas would be areas of the highest strain concentration. This observation was conformed during experimental analysis of the structures after strain application under the SEM. The SEM images show fracture occurring in all three coating thicknesses under strain. However, upon releasing the strain it was expected that the coatings would behave differently, and it was hypothesized that the fracture composition of the $33 \mathrm{~nm}$ coating would provide clues to its behavior. Figure $4 \mathrm{~d}$ shows the EDS data from the performed experiment. It is observed that, among all three coating thicknesses, gold is abundantly present outside the fracture point under strain. The significant differences occur when the EDS is performed inside the microfracture areas. While peaks for oxygen and carbon (denoting the resin underneath the gold) are observed in all three coatings, only the $33 \mathrm{~nm}$ thickness shows a significant amount of gold inside the micro-fracture region, relative to the other two coatings. This could explain the lower area under such a hysteresis curve for this coating. 


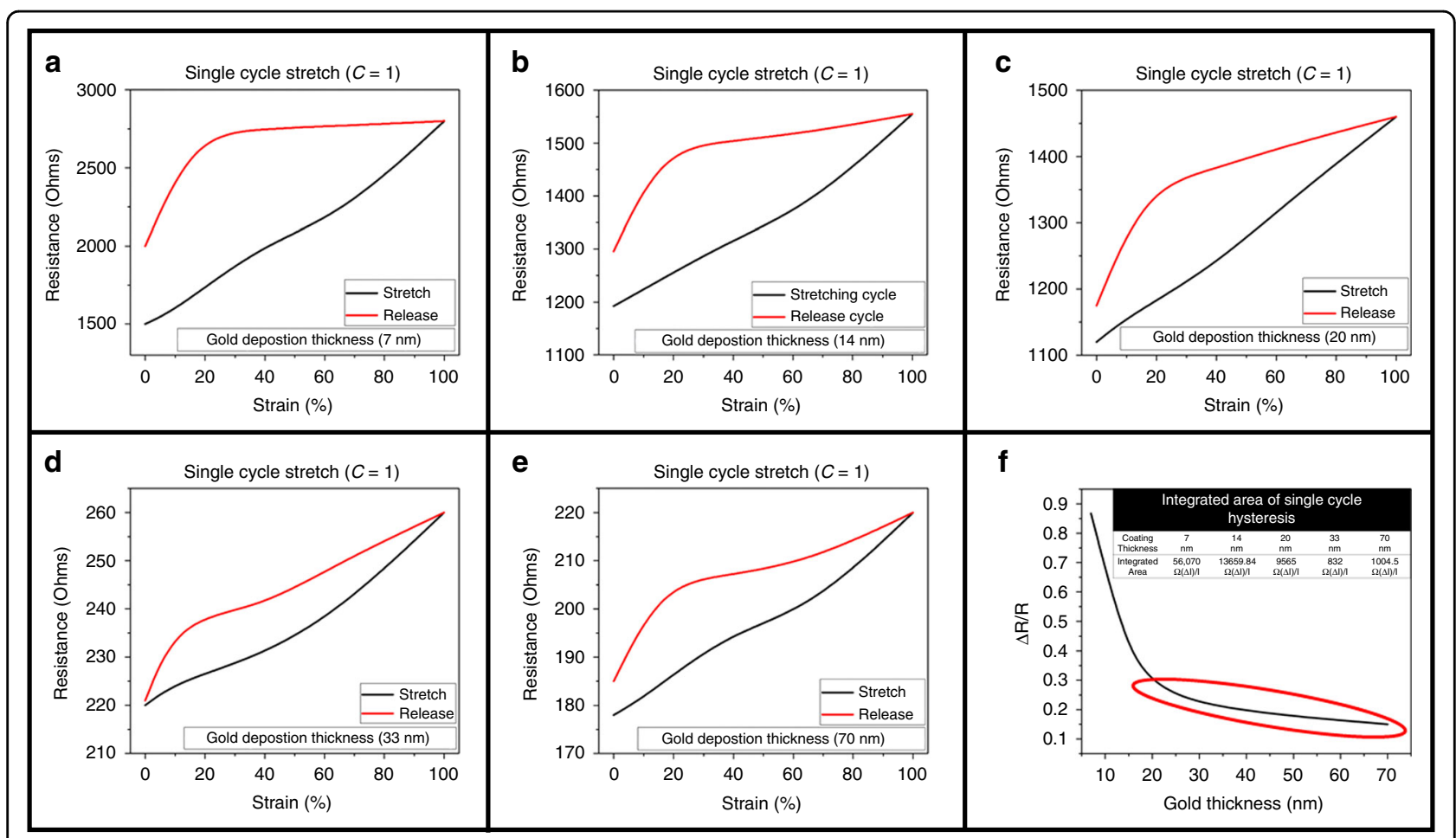

Fig. 3 Single cycle hysteresis (strain and release) graphs for each of the Au coating thicknesses. a $7 \mathrm{~nm}$ coating hysteresis cycle. $\mathbf{b} 14 \mathrm{~nm}$ coating hysteresis cycle. c $20 \mathrm{~nm}$ coating hysteresis cycle. $\mathbf{d} 33 \mathrm{~nm}$ coating hysteresis cycle. e $70 \mathrm{~nm}$ coating hysteresis cycle. f $\Delta R / R$ for each of the coating thicknesses. The red highlighted area indicated coatings that are more suitable for consistent conduction performance over strain, including the 20, 33, and $70 \mathrm{~nm}$ coatings. The inset table lists the tabulated hysteresis integration areas, indicating the most ( $33 \mathrm{~nm}$ ) and least ( $7 \mathrm{~nm}$ ) consistent conduction performance over the single cycle.

The aluminum peak in the EDS data is attributed to the background metal spectrum of the sample stage. Figure $4 \mathrm{e}$ is an SEM image, illustrating the sharp micro-fracture occurring in the $70 \mathrm{~nm}$ coating, which could explain the increased resistance observed once at the end of the strain application cycle in Fig. 3.

In order to obtain fatigue limits of these coatings on the optimized $\mu$ serpentines, repeated strains were applied to the $33 \mathrm{~nm}$ and $70 \mathrm{~nm}$ coated $\mu$ serpentines. Figure $5 \mathrm{a}$, b show the strain and release profiles for the $33 \mathrm{~nm}$ and $70 \mathrm{~nm}$ coatings ( $C=3$ cycles), respectively. These results are similar to a single-cycle results observed in Fig. 3 . The three-cycle hysteresis curve shown in Fig. $5 \mathrm{c}$ illustrates irregularities observed in the $70 \mathrm{~nm}$ coated $\mu$ serpentines. More than half of the tests performed at this thickness contain seemingly random spiking activity during any of its strain cycles. As mentioned previously in the EDS data of Fig. $4 \mathrm{~d}$ and the associated SEM images of Fig. 4c, e, the $70 \mathrm{~nm}$ coating separates dramatically during strain, which directly contributes to the spiking seen in these cycles. Fatigue limit of both the 33- and the $70 \mathrm{~nm}$ coating on the optimized $\alpha=$ $10^{\circ} \mu$ serpentine is depicted in Fig. $5 \mathrm{~d}$. The $70 \mathrm{~nm}$ coating is observed to fail under 30 strain cycles, whereas the $33 \mathrm{~nm}$ coating continues to perform up to 60 cycles of strain indicating approximately double the fatigue limit. These observed results were the rationale to pursue the $33 \mathrm{~nm}$ coating, which demonstrated a more reliable performance.

Figure 6 demonstrates the electrical characterization through resistance measurements of the $\mu$ serpentine base structure under twisting, bending, and conformation. Figure 6a shows the tight resistance distribution (mean of $275 \Omega ;+/-10.0 \Omega$ ) of the optimized $\mu$ serpentine design as it is twisted for $N=11$ cycles prior to becoming unusable. Figure $6 \mathrm{~b}$ shows the slight variance of the resistance values (mean of $278 \Omega ;+/-8.62 \Omega$ ) during $N=5$ twist cycles and the subsequent $N=5$ turns to untwist the $\mu$ serpentine (mean of $260 \Omega ;+/-1.82 \Omega$ ).

The variance from higher twisting to untwisting values is attributable to the relaxation of the gold coating and further demonstrates the reliability of the 33-nm coating. Figure $6 \mathrm{c}$ shows the resistance values (mean of $282 \Omega ;+/-0.88 \Omega$ ) for the cuff conformation of the $\mu$ serpentine device, and Fig. $6 \mathrm{~d}$ shows a very similar and tight grouping for the resistance values for an end-to-end bending conformation (mean of $282 \Omega ;+/-1.16 \Omega$ ). The images in Fig. $6 \mathrm{e}-\mathrm{g}$ are micrographs of the twisting and bending conformations performed, with Fig. 6e representing data shown in Fig. 6a, b, Fig. 6 f representing Fig. 6c, and Fig. 6g paired with Fig. 6d. 


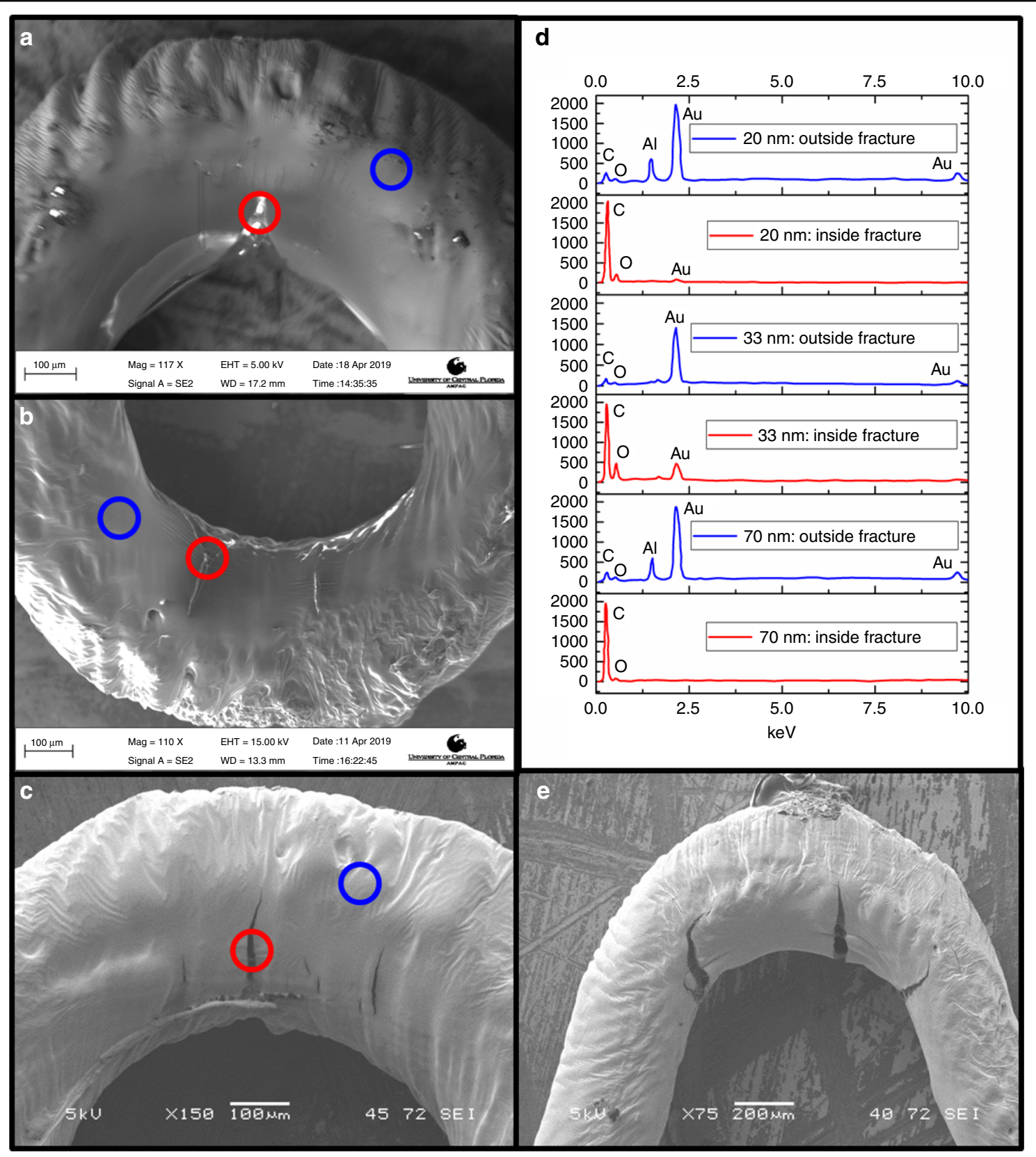

Fig. 4 SEM and associated EDS data for analysis of optimized Au coating thickness after the application of uniaxial strain. In all the SEM images, the circles indicate points where EDS analysis was performed, with red being inside fracture points and blue being outside fracture points. a SEM of the $20 \mathrm{~nm}$ coating for EDS analysis. $\mathbf{b}$ SEM of the $33 \mathrm{~nm}$ coating for EDS analysis. c SEM of the $70 \mathrm{~nm}$ coating for EDS analysis. $\mathbf{d}$ Color corresponding EDS data for red and blue circles from each of the previous SEM images. Generally, more Au was located outside of the fracture as was expected, and the real difference in the analysis was the amount of Au that remained in the fracture. e SEM image of a separate $70 \mathrm{~nm}$-thick Au coating on a $\mu$ serpentine after the application of strain, demonstrating much large fracturing of the Au film.

The stability of the resistance in all of these conformations further lends credence to $\mu$ serpentine optimization for a microelectrode application.

Figure 7 demonstrates the fully integrated and assembled 3D printed, $\mu$ serpentine 3D microelectrodes for biosensor applications. Figure $7 \mathrm{a}$ shows the design to device translation, representing a highly controllable and repeatable fabrication process. Figure $7 \mathrm{~b}-\mathrm{d}$ depict the flexibility of this fully encapsulated device (Kapton package and PDMS insulation) in several key conformations: twisting (Fig. 7b), cuff (Fig. 7c), and outward end-toend bending (Fig. $7 \mathrm{~d}$ ). The device was robust enough to 


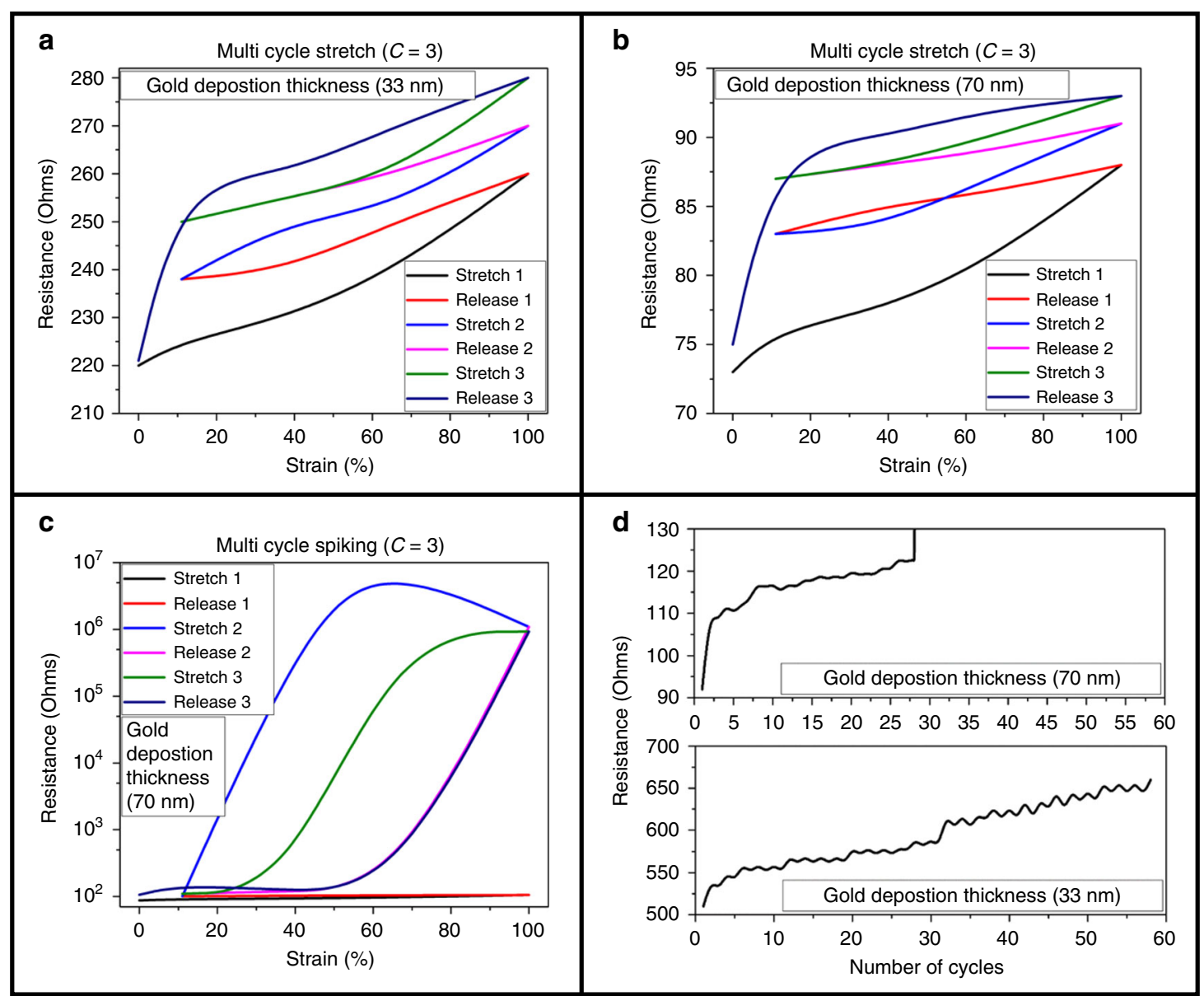

Fig. 5 Multicycle hysteresis and reliability strain performance. a, b Multicycle hysteresis of the $33 \mathrm{~nm}(\mathbf{a})$ and $70 \mathrm{~nm}$ (b) Au coatings on the $\mu$ serpentine. Looking only at these graphs, the performance of both coatings is similar. When examining the larger film fracturing samples from Fig. 4 e, a large strain spiking signature can be seen (c). $\mathbf{d}$ Reliability measurements during fatigue testing for both the 33 - and $70 \mathrm{~nm}$ Au coatings, further demonstrating the choice of $33 \mathrm{~nm}$ as an optimized coating thickness for this work.

recover its original shape immediately, after the application of these flexural strains. The fabrication can be adapted potentially with a PDMS package and liquid $\mathrm{EInGaN}^{35}$ or $\mathrm{CPDMS}^{36}$ metallic traces to achieve a fully stretchable biosensor. As an initial step in device demonstration, Kapton ${ }^{\oplus}$ was chosen due to its thermal stability, ease of defining through laser-micromachining, and good adhesion properties to deposited metal traces ${ }^{37}$. PDMS has been experimentally observed by our group, to be inhibited from curing at the interface between the resin and the elastomer. However, through the metallization of the $3 \mathrm{D}$ printed $\mu$ serpentine (a necessary step in the device microfabrication), no issues with curing the PDMS layer on the 3D printed resin were observed. Figure 7e shows an SEM overview of the fully encapsulated and fabricated microelectrode device. The uninsulated electrode tips can be observed emerging from the PDMS, while the underlying $\mu$ serpentine structure can also be observed owing to the conformal nature of PDMS coating and casting. The laser micromachined scribe line to isolate the electrodes from one another can similarly be observed. Figure $7 f$ depicts a close-up SEM of the 3D printed, metallized electrode tips that are exposed above the PDMS insulation. Edge of this tip is shown in Fig. $7 \mathrm{~g}$, and the striations of the surface of the exposed tip can be viewed. The striations are formed in this way, due to the optimal $\mu$ SLA printing conditions as detailed in Kundu et al. ${ }^{38}$, in which a $45^{\circ}$ print angle was determined to be ideal for resolving the full electrode towers.

The versatility which $3 \mathrm{D}$ printing imparts on the fabrication process means that complex, arbitrarily defined $3 \mathrm{D}$ structures could be incorporated into the device, leading to a variety of potential lab-on-a-chip, wearable, and cell culture $^{39}$ applications in areas, such as microneedles, 3D microfluidics, cellular constructs, and helices (Fig. S2). Using the fabrication process described in this work and a printer 


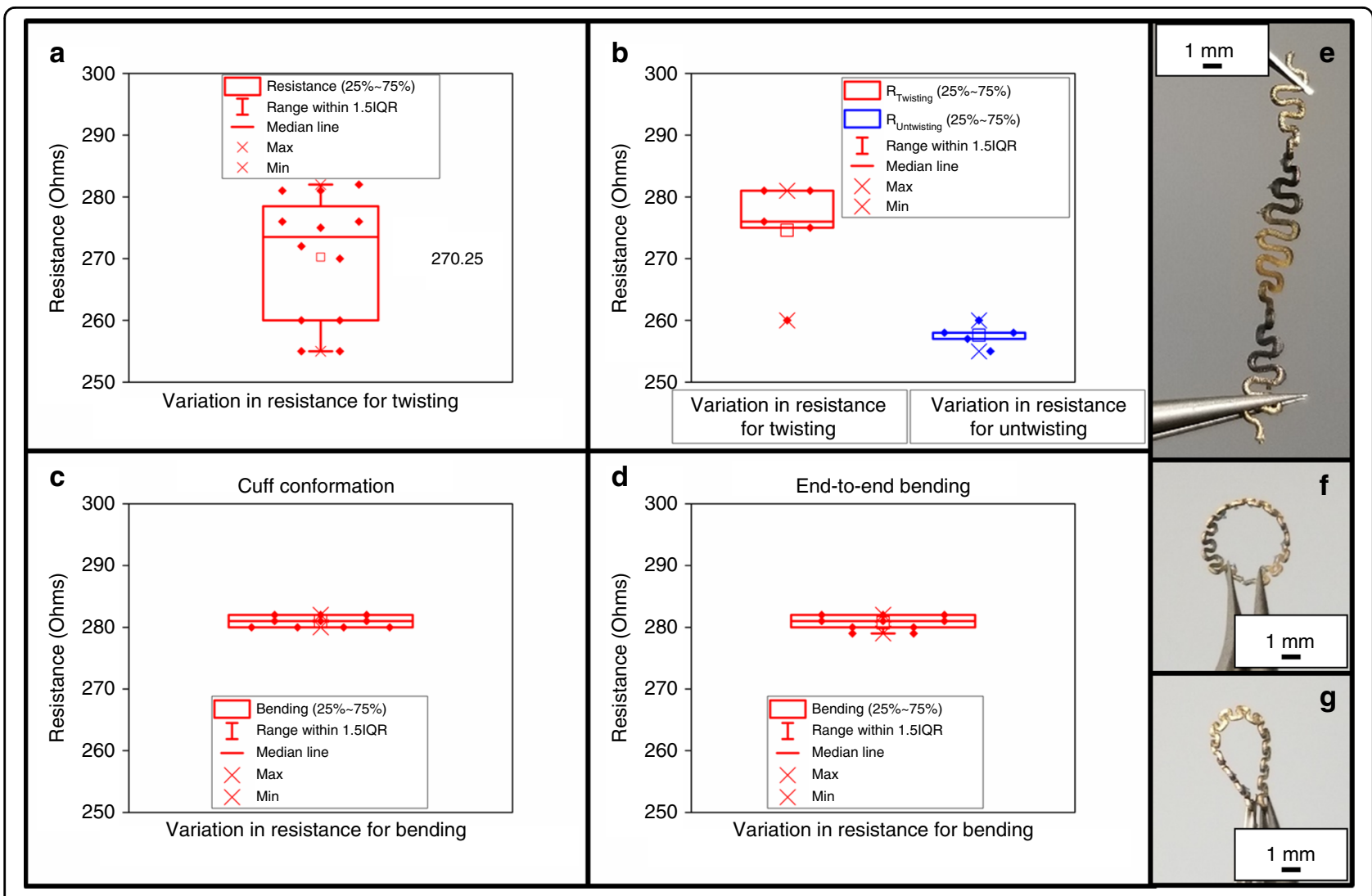

Fig. 6 Conformational bending/twisting analysis of the optimized $\mu$ serpentine with $33 \mathrm{~nm}$ Au coating. a Twisting conformation of the $33 \mathrm{~nm}$ Aucoated $\mu$ serpentine, demonstrating a tight resistance signature for $N=11$ twists. $\mathbf{b}$ A similar twisting signature plot, incorporating resistance values for $N=5$ twisting and untwisting cycles, also demonstrating a tight grouping. c A cuff conformation resistance plot for the $\mu$ serpentine, showing reliable performance after greater than $N=25$ bends. $\mathbf{d}$ End-to-end bending of the $\mu$ serpentine, also demonstrating a very similar tight grouping to $\mathbf{c}$. e Optical image of twisting the $\mu$ serpentine. $\mathbf{f}$ Optical image of the $\mu$ serpentine in a cuff conformation. $\mathbf{g}$ Optical image of the end-to-end bending of the $\mu$ serpentine.

with smaller minimum feature sizes (such as Nanoscribe ${ }^{40}$ or Asiga Digital Light Projection printers ${ }^{41}$ ), smaller functional electrodes could be manufactured, potentially extending various surface geometries into the nanoscale. According to Widlund et al. ${ }^{24}$, the calculations used to optimize the printed $\mu$ serpentines are ubiquitous due to the geometric nature of the key parameters of the device $(l, R, w$, $\alpha$, etc.). However, practical design considerations, printing technique, and usability in packaged biosensor designs control the characteristics of $\mu$ serpentines.

Figure 8 compiles the electrical characterization of the 3D microelectrode $\mu$ serpentine device. Figure 8a shows the full spectrum $(10 \mathrm{~Hz}$ to $10 \mathrm{MHz})$ impedance of the electrode tips $(\sim 300 \mu \mathrm{m}$ in height) before, during, and after bending strain was applied to the devices. At the electrophysiologically relevant frequency of $1 \mathrm{kHz}^{42}$, the real part of the complex impedance was measured to be $4.2 \mathrm{k} \Omega$ (before), $4.6 \mathrm{k} \Omega$ (during), and $5.2 \mathrm{k} \Omega$ (after) $(N=$ $3)$. These values demonstrate comparable $3 \mathrm{D}$ microelectrode characteristics to other reported approaches in literature, such as Guvanesen et al. ${ }^{19}$, where their average $1 \mathrm{kHz}$ value is $7.6 \mathrm{k} \Omega(+/-2.20 \mathrm{k} \Omega)$. 3D printing high aspect ratio structures using $\mu$ SLA processes exaggerates the striations that are visible in Fig. 7g. We anticipate the higher surface area created by these striations lowered the impedance values measured.

Typically to this end, nanomaterial electroplating or electroless plating has been one highly used method for increasing the surface area of microelectrodes, in order to help better extract biologically relevant data ${ }^{43}$. The micro-texturing inherent in 3D printing could aid in impedance reduction for these interfaces. Figure $8 \mathrm{~b}$ represents phase values of full spectrum impedance before, during, and after the application of bending strain. The phase spectra depict characteristically microelectrode profiles ${ }^{44}$, starting as capacitive at phase between $-20^{\circ}$ and $-40^{\circ}$ and gradually becoming more resistive as the frequency increases, and the phase value approaches $0^{\circ}$. The phase data suggest a pattern observed by us $^{45}$ and other microelectrode research$\mathrm{ers}^{46}$, in which the electrical profile of the mid-range frequencies is governed by a double-layer capacitance $\left(C_{D L}\right)$. The electrode-electrolyte interfacial impedance that occurs is dominated by the resistive elements of the 


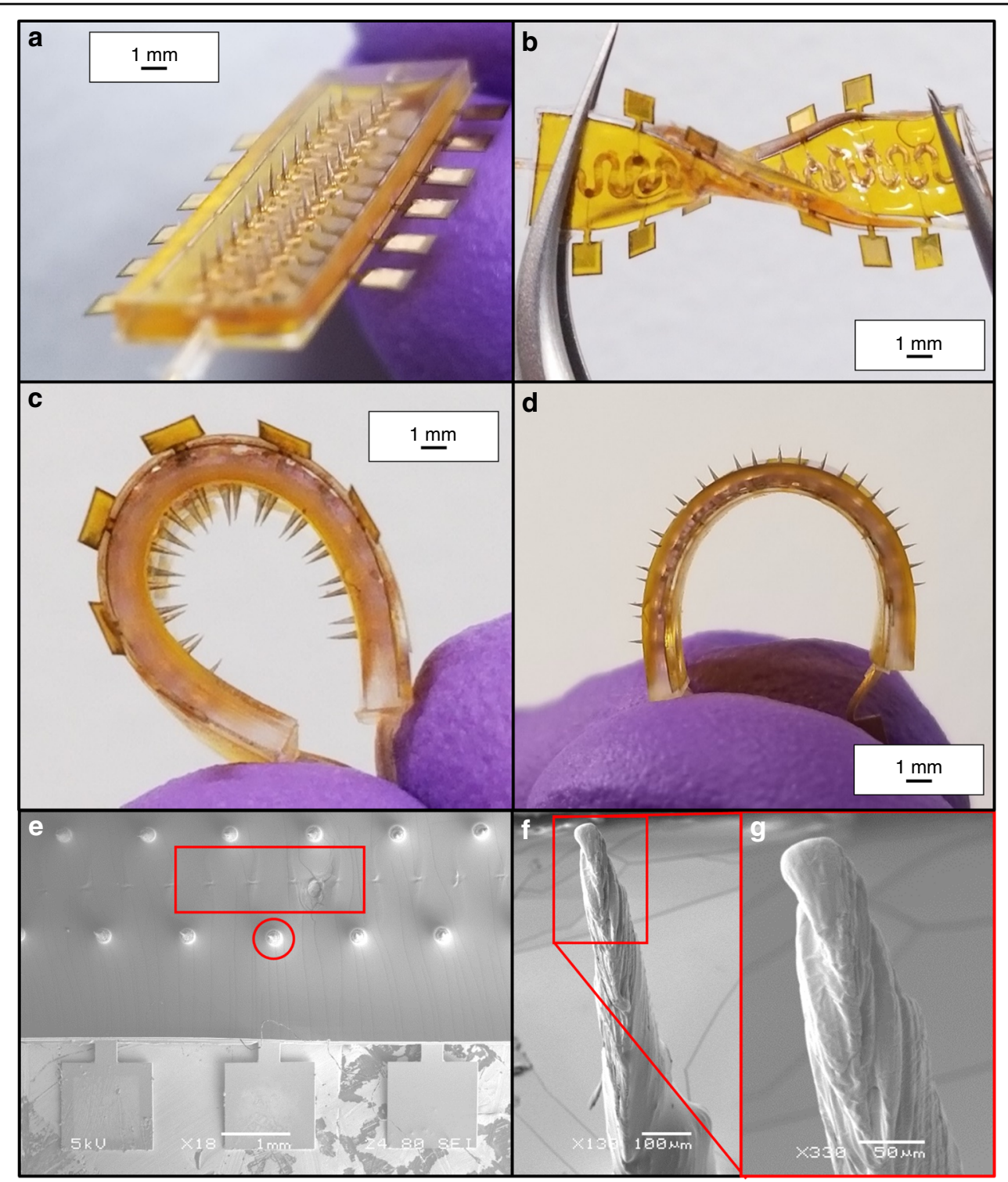

Fig. 7 Optical and SEM images of packaged and assembled $\mu$ serpentine sensor device. a Optical image of the fully assembled 3D microelectrode device, schematically represented in Fig. 1. $\mathbf{b}$ Optical image of the microelectrode $\mu$ serpentine device undergoing twisting with a pair of tweezers. c Optical image of the 3D userpentine microelectrode device undergoing end-to-end bending. $\mathbf{d}$ Optical image of the microelectrode userpentine device in a reverse cuff conformation, exposing the microelectrode needle tips for imaging. e SEM image of the fully assembled device. The highlighted regions denote where the laser isolation trace is located beneath the PDMS layer (and hence are difficult to visualize) and where the exposed circular electrode tips emerge from the PDMS layer. $\mathbf{f}$ SEM image of the exposed electrode tip. After insulation, it is estimated that the electrode tips are $300 \mu \mathrm{m}$ in height above the surface of the PDMS. $\mathbf{g}$ SEM close up of the electrode tip from $\mathbf{f}$, highlighting the naturally formed $\mu S L A$ striations, which contribute to the increased effective surface area of the 3D microelectrode.

phosphate buffer solution at higher frequencies, and as a result slightly more negative phase values are observed. An analytical microelectrode model is fitted to the real and phase parts of the impedance based on the circuit schematic shown in Fig. 8c, which represents the components of a complete $\mathrm{MEA}^{44}$ : solution resistance $\left(R_{\mathrm{S}}\right)$, charge transfer resistance $\left(R_{\mathrm{CT}}\right)$, double-layer capacitance $\left(C_{\mathrm{DL}}\right)$, and the Warburg element $(W)$.
For extraction of the circuit parameters listed, Eq. 3 is solved:

$$
Z(\omega)=R_{\mathrm{S}}+\left[1 /\left(C_{\mathrm{DL}}(\omega)+\left\{1 /\left(R_{\mathrm{CT}}+W(\omega)\right)\right\}\right]\right.
$$

The analytical model fits the experimental data well, demonstrating the impact of the components on the final 


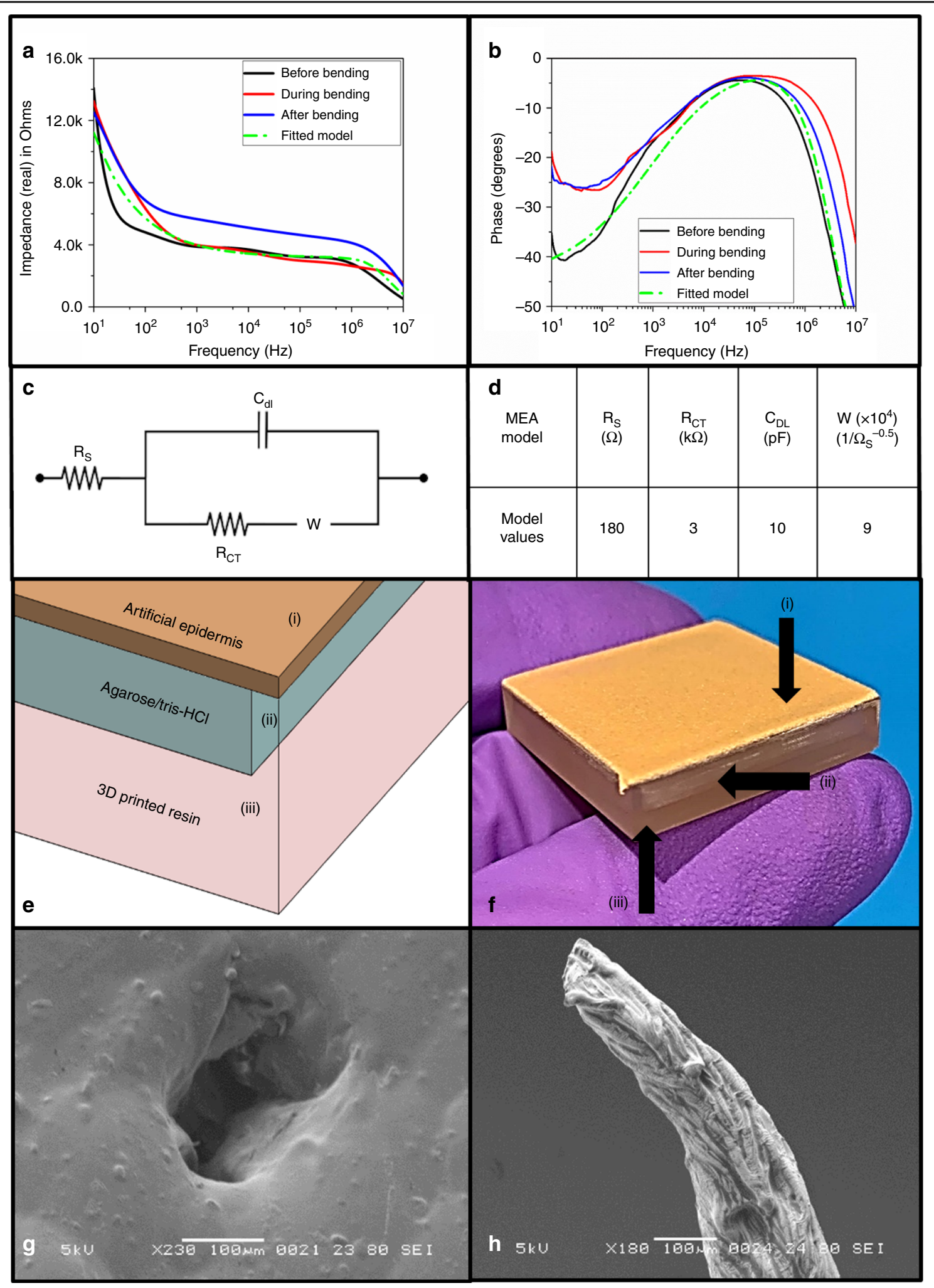

Fig. 8 (See legend on next page.) 
(see figure on previous page)

Fig. 8 Characterization of the microelectrode $\mu$ serpentine device and subsequent artificial epidermis/dermis transdermal electrical recording images. a Full spectrum impedance plot of the device before (black), during (red), and after (blue) bending, with a fitted impedance model (green) from which relevant circuit parameters were extracted. b Full spectrum phase graph for the device before (black), during (red), and after (blue) bending, with a fitted impedance model (green) from which relevant circuit parameters were extracted. c Representative circuit model for the microelectrode array profile extracted from $\mathbf{a}, \mathbf{b}$. $\mathbf{d}$ Extracted circuit parameters from the modeled circuit. e Schematic representation of the artificial skin model: (i) Corresponds to the artificial, non-conductive epidermis patch, (ii) the agarose/Tris-HCl artificial dermis tissue, and (iii) the 3D printed mold accommodating the skin model. $\mathbf{f}$ Optical image of the artificial skin model, with (i-iii) corresponding to the schematic components listed. $\mathbf{g}$ SEM image of a puncture site on the artificial epidermis, demonstrating the feasibility of the 3D printed microneedle microelectrode towers to penetrate skin for potential transdermal/tissue recording applications. $\mathbf{h}$ SEM image of an electrode tip from one device, which was not properly heat cured to give the resin its final robust structure. This is essential, or the resin electrodes will not be able to penetrate the skin or skin model.

electrical profile of the sputter coated, 3D $\mu$ serpentine gold microelectrode. Figure $8 \mathrm{~d}$ contains a table of the extracted values of the equivalent circuit for the analytical model that represents the combined three device states (before, during, and after bending).

In order to demonstrate a wearable 3D MEA sensor application, highlighting the versatility of the $\mu$ serpentine design structure, an artificial skin model was developed in part with the protocol outlined in Besio et al. ${ }^{47}$. The agarose "dermal tissue" was cast in a custom 3D printed mold and covered with a non-conductive epidermal layer, as is represented schematically in Fig. 8e and optically in Fig. 8f. The surface direct current (DC) resistance of the agarose "dermal tissue" model layer was found to be $\sim 60 \mathrm{k} \Omega$ $(+/-1.40 \mathrm{k} \Omega ; N=3)$, and the end-to-end absolute resistance of the penetrated agarose was measured at $30 \mathrm{k} \Omega$ $(+/-0.20 \mathrm{k} \Omega ; N=3)$. The $\mu$ serpentine device electrodes were interfaced with the artificial skin model to record subdermal tissue DC resistance to confirm the ability of the microelectrodes to penetrate the epidermis (Fig. 8g). The device successfully acquired a reading of the underlying tissue model, at DC resistances between 40 and $50 \mathrm{k} \Omega$, across several puncture points in the model. The resistivity of the model according to the resulting DC resistance measurements was calculated to be $\sim 50 \Omega-\mathrm{m}$, well within the expected values ${ }^{47}$. Generally, the $3 \mathrm{D}$ printed resin structures require heat curing after printing to ensure the full stability and rigidity of the fabricated structures ${ }^{38}$. As depicted in Fig. 8h, there was some damage noted to certain microelectrode tips after pressing on the artificial skin model. These tips may not have been completely thermally cured (usually the curing time for other device components serves well for this purpose), but reducing the tower aspect ratio could also help increase their stability after shorter heat curing times. This proof-of-concept demonstration highlights the potential for these sensors to be used in wearable electroencephalogram, electrocardiogram, electromyogram, and nerve conduction measurements ${ }^{47}$.

\section{Materials and methods}

Thin Kapton ${ }^{\oplus}(12.5 \mu \mathrm{m})$ sheets (DuPont ${ }^{\mathrm{TM}}$, USA) were laser micromachined to a size of $20 \mathrm{~mm}$-by- $5 \mathrm{~mm}$, with
$1 \mathrm{~mm}$-by- $1 \mathrm{~mm}$ extensions (6 on either side of the base substrate; 12 total) on which the microelectrode landing pads would be subsequently defined (Fig. 1a(ii)). Laser micromachined shadow masks were machined from $12.5 \mu \mathrm{m}$-thick 316L stainless steel (Trinity Brand Industries, USA) using the QuikLaze 50 ST2 laser micromachining system (Eolite Lasers, USA) with $1064 \mathrm{~nm}$ wavelength infrared (IR) laser light $(6 \mathrm{~mJ}$ power, and $50 \mathrm{~Hz}$ repetition rate) (Fig. 1a(ii)). These masks served as stencils for subsequent metallization. Gold $(5 \mathrm{~N}, 57 \mathrm{~mm}$ by $0.2 \mathrm{~mm}$ Au target; Ted Pella, INC., USA) was deposited on the 3D printed substrate (deposition voltage: $20 \mathrm{mV}$, and $13 \mathrm{~nm} / \mathrm{min}$ deposition rate) to form $33 \mathrm{~nm}$-thick packaging traces and landing pads through the Quorum Q150T Plus sputtering system (Quorum Technologies Ltd., UK) (Fig. 1a(ii)).

The 3D $\mu$ serpentines with and without out-of-plane biosensor structures were designed with the Solidworks 2018 3D CAD software (Dassault Systems, USA) and 3D printed using commercially available clear (FLGPCL04) resins on the Formlabs Form $2 \mu$ SLA 3D printer (Formlabs, USA) (Fig. 1a, b(i)). The $\mu$ serpentines were designed to be $400 \mu \mathrm{m}$-thick, and the pitch between the central points of the U-bends varies between the three printed designs, which are shown in Fig. 2c. The $\alpha=0^{\circ}$ design has a pitch of $1.5 \mathrm{~mm}$; the $\alpha=-33^{\circ}$ design has a pitch of $2.19 \mathrm{~mm}$, and the $\alpha=10^{\circ}$ design has a pitch of $1.3 \mathrm{~mm}$ (Fig. 2c(i-iii)). All out-of-plane 3D printed structures were designed to be $400 \mu \mathrm{m}$ at the base and with a height of $2 \mathrm{~mm}$. The physical printed electrode cones before insulation resolved at approximately $1.1 \mathrm{~mm}$ above the $\mu$ serpentine U-bend, and so the resolved electrode cones before insulation were $400 \mu \mathrm{m}$ at the base and $1.1 \mathrm{~mm}$ in height. The pitch between the 3D structures was designed to be $1.3 \mathrm{~mm}$ (similar to the $\alpha=10^{\circ}$ design). The printed $\mu$ serpentine was similarly metallized utilizing a sputter coater (Quorum Q150T Plus; Quorum Technologies Ltd., UK) with a layer of Gold $(5 \mathrm{~N}, 57 \mathrm{~mm}$ by $0.2 \mathrm{~mm}$ Au target; Ted Pella, INC., USA), under the same deposition rates as outlined above $(20 \mathrm{mV}$, and $13 \mathrm{~nm} /$ min deposition rate) to form conformal coatings across the entire 3D $\mu$ serpentine structure with thicknesses 
ranging from 7 to $70 \mathrm{~nm}$ (Fig. 1a(iii)). The microelectrodes on the $\mu$ serpentines were isolated down the center (both front and backsides) of the structure using the QuikLaze 50 ST2 laser micromachining system (Eolite Lasers, USA) with $1064 \mathrm{~nm}$ wavelength IR mode $(6 \mathrm{~mJ}$ power, and $50 \mathrm{~Hz}$ repetition rate), which selectively ablated the gold and did not damage the resin (Fig. 1a(iii)). For the MEA design, the $\mu$ serpentines were aligned with the terminated metal traces of the packaging substrate, and a small droplet of the Epo-tek EJ2189 silver-ink (Epo-Tech, USA) was placed on the $\mu$ serpentines/trace interface to ensure connectivity. The package was allowed to cure for $24 \mathrm{~h}$ at $45^{\circ} \mathrm{C}$ (Fig. 1a(iii)).

A drop-casted layer of 10:1 PDMS bulk polymer to curing agent (Slygard-184, Dow Corning, USA) was defined as the final insulation layer on the 3D electrodes (Fig. 1a(iii)). A custom designed and 3D printed mold was developed to assemble the devices into their final form factor. PDMS was cast within this mold to ensure a uniform thickness across the device. The assembly mold was sputter coated with gold separation layer to a thickness of $70 \mathrm{~nm}$, to ensure curing of PDMS, which was observed by our group, to be inhibited by the resin. The thickness of the final PDMS insulation was defined and insulated at 1$\mathrm{mm}$ thick (Fig. 1a(iv)). The device assembly was cured at $50^{\circ} \mathrm{C}$ for an additional $24 \mathrm{~h}$ to attain the full crosslinked mechanical properties of the PDMS ${ }^{48}$.

Elongation experiments for $\mu$ serpentine characterization were performed by clipping contacts to both ends of the $\mu$ serpentines and recording the DC resistance measurements from a Keithley 2400 Sourcemeter (Tektronix, USA) (Figs. 3, 5, and 6). Twisting/bending analysis and hysteresis cycling were performed with tweezers under a stereoscope with wire leads epoxied to the landing pads for electrical characterization during the application of strain. Full-spectrum impedance measurements were performed with a BODE 100 Impedance Analyzer (Omicron Labs, Austria) with a Platinum (Pt) anode in Dulbecco's Phosphate Buffered Saline (1x) (Gibco, USA; Fig. $8 \mathrm{a}, \mathrm{b})$. For SEM imaging of samples, the Zeiss Ultra-55 SEM (Zeiss, Germany) was used, and EDS was performed on the same SEM with the Noran System 7 EDS with Silicon Drift Detector X-ray Detector (Thermo Fisher Scientific, USA) (Figs. 2, 4, 7, and 8). All optical images were obtained with an iPhone XS (Apple, USA). Data graphing was performed in Origin 2016 (OriginLab Corporation, USA). Data fitting and impedance modeling were performed in MATLAB R 2018b (Mathworks, USA). Effective stiffness and normalized maximum tensile strain calculations were defined and implemented with appropriate design values using Wolfram Mathematica 11.3 (Wolfram, USA) (Fig. 2a, b). The Agarose dermal tissue model was created from a $1 \mathrm{M}$ solution of Tris- $\mathrm{HCl}(\mathrm{pH}$
6.1) and powdered agarose as per Besio et al. ${ }^{47}$. The mixture was placed in a beaker and stirred continuously to a boil of $100^{\circ} \mathrm{C}$ on a hotplate. According to the refs. ${ }^{47,49,50}, 3 \mathrm{~mm}$-thick agarose mixed in the aforementioned protocol models dermal tissue closely, with a conductivity of $0.06 \mathrm{~S} / \mathrm{m}$. The agar was poured into a custom 3D printed $25 \mathrm{~mm}$-by- $25 \mathrm{~mm}$ mold ( $3 \mathrm{~mm}$-thick as per the protocol from these papers) and allowed to fully crosslink at $25^{\circ} \mathrm{C}$ for an hour. The epidermis was modeled with an artificial, $500 \mu \mathrm{m}$-thick epidermal patch (Remedy Simulation Group, USA) and attached to the mold/agarose dermis with integrated adhesive. The artificial epidermal patch is non-conducting to model an enhanced effect of the dead skin layer of the stratum corneum present in real epidermal skin layers. 3D MEAs (depicted in Fig. 7a) were pressed onto the artificial epidermis/ dermis skin model and DC resistance values were obtained from the 3D electrodes across the device using a Keithley 2400 Sourcemeter (Tektronix, USA) (Fig. 8f).

\section{Conclusions}

We have explored the capabilities and limitations of 3D $\mu S L A$ printed $\mu$ serpentine for their applicability to $3 \mathrm{D}$ microelectrodes. We have further integrated such metallized $\mu$ serpentines with a Kapton ${ }^{\circledR}$ package and a PDMS insulated to develop a dynamic 3D MEA. The $\mu$ serpentine base structures used were optimized according to two key compound equations for the effective stiffness and maximum U-bend strain. The final optimized $\mu$ serpentines had an $l / R$ ratio of 2 , and an $\alpha$ of $10^{\circ}$, creating a $\mu$ serpentines that could stretch up to $155 \%$ its resting length. This optimized $\mu$ serpentine was subsequently characterized with varying conformal gold coating thicknesses, to find the optimal thickness to retain resistance values during strain. The resulting coating thickness was found to be $33 \mathrm{~nm}$ and performed equally as well over twisting and bending strain analyses and with good reliability over 60 strain cycles. The final optimized and coated $\mu$ serpentine structure was integrated into a device package built on PI (Kapton $\left.{ }^{\circledR}\right)$ substrates with metallized traces to connect to the laser isolated 3D microelectrode and encapsulated with PDMS insulation. The 3D microelectrode device was characterized for impedance and phase over a full frequency spectrum $(10 \mathrm{~Hz}$ to $10 \mathrm{MHz})$, and the resulting electrophysiologically relevant $1 \mathrm{kHz}$ values were measured for a dynamic MEA application: $4.2 \mathrm{k} \Omega$ (before bending), $4.6 \mathrm{k} \Omega$ (during bending), and $5.2 \mathrm{k} \Omega$ (after bending). This device was then employed to procure transdermal readings across an artificial agarose skin model, measuring the expected resistivity of $50 \Omega-\mathrm{m}$. This details the capabilities, limitations, and the versatility of $\mu$ SLA printed serpentine-based 3D microstructures for various 
sensor devices with applications in wearable technologies, as well as dynamic cell culturing and in vitro conditions.

\section{Acknowledgements}

The authors would like to acknowledge and thank the University of Central Florida for providing start-up funding to the corresponding author that funded this effort. The authors would also like to thank the Nanoscience Technology Center and the Materials Characterization Facility at the University for usage of their equipment.

\section{Author details}

${ }^{1}$ Nanoscience Technology Center (NSTC), University of Central Florida, Orlando, FL 32826, USA. ${ }^{2}$ Burnett School of Biomedical Sciences, University of Central Florida, Orlando, FL 32827, USA. ${ }^{3}$ Department of Materials Science \& Engineering, University of Central Florida, Orlando, FL 32816, USA. ${ }^{4}$ Department of Electrical \& Computer Engineering, University of Central Florida, Orlando, FL 32816, USA

\section{Author contributions}

C.D. conceived the device design and the decision to investigate using serpentine architectures for this purpose. C.D. and S.R. designed the experimental plan for the device. C.D. and A.K. performed the experimental work and plotted the data, and A.K. fit the impedance model with an equivalent circuit. C.D. wrote the manuscript, and all authors contributed to the final editing of the manuscript.

\section{Conflict of interest}

The authors declare that they have no conflict of interest.

Supplementary information accompanies this paper at https://doi.org/ 10.1038/s41378-019-0129-3.

Received: 9 July 2019 Revised: 16 October 2019 Accepted: 18 November 2019

Published online: 20 April 2020

\section{References}

1. Amjadi, M., Kyung, K.-U., Park, I. \& Sitti, M. Stretchable, skin-mountable, and wearable strain sensors and their potential applications: a review. Adv. Funct. Mater. 26, 1678-1698 (2016).

2. Mukhopadhyay, S. C. Wearable sensors for human activity monitoring: a review. IEEE Sens. J. 15, 1321-1330 (2015).

3. Chang, T. H. et al. Stretchable graphene pressure sensors with Shar-Pei-like hierarchical wrinkles for collision-aware surgical robotics. ACS Appl. Mater. Interfaces 11, 10226-10236 (2019).

4. Pang, C., Lee, C. \& Suh, K.-Y. Recent advances in flexible sensors for wearable and implantable devices. J. Appl. Polym. Sci. 130, 1429-1441 (2013).

5. Lipomi, D. J. et al. Skin-like pressure and strain sensors based on transparent elastic films of carbon nanotubes. Nat. Nanotechnol. 6, 788 (2011).

6. $\mathrm{Xu}, \mathrm{S}$. et al. Stretchable batteries with self-similar serpentine interconnects and integrated wireless recharging systems. Nat. Commun. 4, 1543 (2013).

7. Hopcroft, M. A., Nix, W. D. \& Kenny, T. W. What is the Young's modulus of silicon? J. Microelectromech. Syst. 19, 229-238 (2010).

8. Rho, J. Y., Ashman, R. B. \& Turner, C. H. Young's modulus of trabecular and cortical bone material: ultrasonic and microtensile measurements. J. Biomech. 26, 111-119 (1993).

9. Gleskova, H. et al. Field-effect mobility of amorphous silicon thin-film transistors under strain. J. Non-Cryst. Solids 338-340, 732-735 (2004).

10. Sun, Y. et al. Printed arrays of aligned GaAs wires for flexible transistors, diodes, and circuits on plastic substrates. Small 2, 1330-1334 (2006).

11. Lv, C., Yu, H. \& Jiang, H. Archimedean spiral design for extremely stretchable interconnects. Extrem. Mech. Lett. 1, 29-34 (2014).

12. Pan, T. et al. Experimental and theoretical studies of serpentine interconnects on ultrathin elastomers for stretchable electronics. Adv. Funct. Mater. 27, 1702589 (2017).

13. Wang, S., Huang, Y. \& Rogers, J. A. Mechanical designs for inorganic stretchable circuits in soft electronics. IEEE Trans. Compon. Packaging Manuf. Technol. 5, 1201-1218 (2015).
14. Stoppa, M. \& Chiolerio, A. Wearable electronics and smart textiles: a critical review. Sensors 14, 11957-11992 (2014).

15. Liu, Y., Pharr, M. \& Salvatore, G. A. Lab-on-skin: a review of flexible and stretchable electronics for wearable health monitoring. ACS Nano 11, 9614-9635 (2017)

16. Kim, R., Joo, S., Jung, H., Hong, N. \& Nam, Y. Recent trends in microelectrode array technology for in vitro neural interface platform. Biomed. Eng. Lett. 4, 129-141 (2014).

17. Rajaraman, S. et al. Metal-transfer-micromolded three-dimensional microelectrode arrays for in-vitro brain-slice recordings. J. Microelectromech. Syst. 20, 396-409 (2011).

18. Yao, S. et al. A wearable hydration sensor with conformal nanowire electrodes Adv. Healthc. Mater. 6, 1601159 (2017).

19. Guvanasen, G. S. et al. A stretchable microneedle electrode array for stimulating and measuring intramuscular electromyographic activity. IEEE Trans. Neural Syst. Rehabil. Eng. 25, 1440-1452 (2017).

20. Gardner, R. D., Zhou, A. \& Zufelt, N. A. Development of a microelectrode array sensing platform for combination electrochemical and spectrochemical aqueous ion testing. Sens. Actuators B Chem. 136, 177-185 (2009).

21. Ni, Y. et al. A review of 3D-printed sensors. Appl. Spectrosc. Rev. 52, 623-652 (2017).

22. Hart, C. et al. Rapid nanofabrication of nanostructured interdigitated electrodes (nIDEs) for long-term in vitro analysis of human induced pluripotent stem cell differentiated cardiomyocytes. Biosensors (Basel) 8 , 88 (2018).

23. Formlabs. Safety Data Sheet for Formlabs Clear Resin. https://archive.media. formlabs.com/upload/Clear_Resin_SDS_EU.pdf (2018).

24. Widlund, T., Yang, S., Hsu, Y.-Y. \& Lu, N. Stretchability and compliance of freestanding serpentine-shaped ribbons. Int. J. Solids Struct. 51, 4026-4037 (2014).

25. DuPont. Summary of Properties of Kapton Polyimide. https://www. dupont.com/content/dam/dupont/products-and-services/membranesand-films/polyimdefilms/documents/DEC-Kapton-summary-ofproperties.pdf (2019).

26. Armani, D., Liu, C. \& Aluru, N. Re-configurable fluid circuits by PDMS elastomer micromachining. In Technical Digest. IEEE International MEMS 99 Conference. Twelfth IEEE International Conference on Micro Electro Mechanical Systems https://doi.org/10.1109/MEMSYS.1999.746817 (IEEE, 1999).

27. Johnston, I. D., McCluskey, D. K., Tan, C. K. L. \& Tracey, M. C. Mechanica characterization of bulk Sylgard 184 for microfluidics and microengineering. J. Micromech. Microeng. 24, 035017 (2014).

28. Du, P., Lin, X. \& Zhang, X. Tunable electrical and mechanical responses of PDMS and polypyrrole nanowire composites. J. Phys. D Appl. Phys. 46, 195303 (2013).

29. Liu, Y. L. et al. Stretchable electrochemical sensor for real-time monitoring of cells and tissues. Angew. Chem. Int. Ed. Engl. 55, 4537-4541 (2016).

30. Irgens, F. Continuum Mechanics, 1st edn 199-301 (Springer-Verlag, Berlin, Heidelberg, 2008).

31. Yang, S., Qiao, S. \& Lu, N. Elasticity solutions to nonbuckling serpentine ribbons J. Appl. Mech. 84, 021004 (2016).

32. Bangs Laboratories. I. Material Properties, Tech support Doc 0021. https:// www.bangslabs.com/sites/default/files/imce/docs/TSD\%200021\%20Material\% 20Properties\%20Web.pdf (2015).

33. SCIENCES, E. M. Technical Data Sheets: Sputter Coater Principles. https://www.emsdiasum.com/microscopy/technical/datasheet/ sputter_coating.aspx (2019).

34. Kong, J.-H., Jang, N.-S., Kim, S.-H. \& Kim, J.-M. Simple and rapid micropatterning of conductive carbon composites and its application to elastic strain sensors. Carbon 77, 199-207 (2014).

35. Mengüç, Y. et al. Soft wearable motion sensing suit for lower limb biomechanics measurements. In 2013 IEEE International Conference on Robotics and Automation 5309-5316 (ICRA, 2013).

36. Tavakoli, M. et al. Carbon doped PDMS: conductance stability over time and implications for additive manufacturing of stretchable electronics. J. Micromech. Microeng. 27, 035010 (2017).

37. DUPONT. Kapton: summary of properties. https:/www.dupont.com/content/ dam/dupont/products-and-services/membranes-and-films/polyimde-films/ documents/DEC-Kapton-summary-of-properties.pdf (2018).

38. Kundu, A., Ausaf, T. \& Rajaraman, S. 3D printing, ink casting and micromachined lamination (3D PICLmuM): a makerspace approach to the 
fabrication of biological microdevices. Micromachines (Basel) https://doi.org/ 10.3390/mi9020085 (2018)

39. Hermanstyne, T. O., Granados-Fuentes, D., Mellor, R. L., Herzog, E. D. \& Nerbonne, J. M. Acute knockdown of Kv4. 1 regulates repetitive firing rates and clock gene expression in the suprachiasmatic nucleus and daily rhythms in locomotor behavior. eNeuro https://doi.org/10.1523/ENEURO.0377-16.2017 (2017).

40. Nanoscribe. Photonic Professional GT2: Highest Resolution 3D Printing. https://www.nanoscribe.com/fileadmin/Nanoscribe/Solutions/ Photonic_Professional_GT2/PhotonicProfessionalGT2.pdf (2019).

41. Asiga. Asiga Max X UV27 Spec Sheet. https://www.asiga.com/products/ printers/max_series/max_X/ (2019).

42. Azim, N. et al. Precision plating of human electrogenic cells on microelectrodes enhanced with precision electrodeposited nanoporous platinum for cell-based biosensing applications. J. Microelectromech. Syst. 28, 50-62 (2019).

43. Kundu, A. et al. Optimization of makerspace microfabrication techniques and materials for the realization of planar, 3D printed microelectrode arrays in under four days. RSC Adv. 9, 8949-8963 (2019).
44. Franks, W., Schenker, I., Schmutz, P. \& Hierlemann, A. Impedance characterization and modeling of electrodes for biomedical applications. IEEE Trans. Biomed. Eng. 52, 1295-1302 (2005).

45. Azim, N. et al. Fabrication and characterization of a 3D printed, microelectrodes platform with functionalized electrospun nano-scaffolds and spin coated 3D insulation towards multi-functional biosystems. J. Microelectromech. Syst. 28, 606-618 (2019).

46. Wang, R. et al. Fabrication and characterization of a parylene-based threedimensional microelectrode array for use in retinal prosthesis. J. Microelectromech. Syst. 19, 367-374 (2010).

47. Prasad, W. B. A. Analysis of skin-electrode impedance using concentric ring electrode. Conf. Proc. IEEE Eng. Med. Biol. Soc. 1, 6414-6417 (2006).

48. Schneider, F., Fellner, T., Wilde, J. \& Wallrabe, U. Mechanical properties of silicones for MEMS. J. Micromech. Microeng. 18, 065008 (2008).

49. Peters, J. \& G. S. M. H. M Estimation of the electrical conductivity of human tissue. Electromagnetics 21, 545-557 (2001).

50. Yamamoto, T. \& Yamamoto, Y. Electrical properties of the epidermal stratum corneum. Med. Biol. Eng. 14, 151-158 (1976). 